\title{
Une «Journée de réflexion» c'est bien, mais ce n'est pas suffisant
}

\section{Werner Bauer}

Dr, Président de I'Institut suisse pour la formation postgraduée et continue (ISFM)

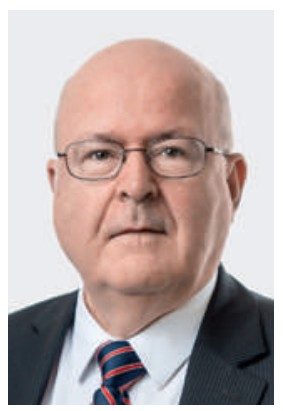

Le rédacteur en chef du Bulletin des médecins suisses, Bruno Kesseli, revient en page 376 sur la «Journée de réflexion» organisée conjointement par l'Institut suisse pour la formation postgraduée et continue (ISFM) et le Collège des Doyens des facultés de médecine. Cette rencontre a été l'occasion bienvenue de débattre des enjeux et des défis de la formation médicale, d'esquisser des projets et de rechercher des solutions.

Jusque-là, très bien, c'est important! Mais une remarque entendue plusieurs fois lors de cette journée interpelle. Il s'agit de la place accordée à l'enseigne-

La formation postgraduée représente beaucoup d'investissement. Par conséquent, elle doit être reconnue, également sur le plan financier.

ment pour la formation postgraduée à l'hôpital. Les représentants des hôpitaux universitaires ont notamment déploré que de nombreux médecins cadres considèrent de plus en plus le «teaching» comme une charge supplémentaire qui s'ajoute aux soins aux patients, aux travaux scientifiques ou aux tâches administratives. Par conséquent, beaucoup renoncent malheureusement à un perfectionnement pédagogique et ne consacrent que le temps minimum à la formation postgraduée, qu'elle soit pratique ou théorique. Tant que les cours proposés seront facultatifs, ils susciteront peu l'intérêt des médecins cadres. Dans les petits hôpitaux en revanche, le contact direct et la proximité contribuent à un meilleur climat pour le transfert des connaissances, ce que confirment les enquêtes réalisées auprès des médecins-assistants.

Rappelons cependant que les hôpitaux universitaires regroupent près de $35 \%$ des postes de formation postgraduée. Dans ces grands centres, comme l'a formulé la directrice d'une clinique, «le formateur devrait être revalorisé», ainsi que son activité d'enseignement qui devrait devenir un critère pour l'évolution de la carrière. Dans ce contexte, il s'agit donc de réfléchir en termes de motivation, d'incitatifs efficaces et, peut-être aussi, d'obligation.

Il est bien évident que le financement de la formation postgraduée, pour lequel aucune solution satisfaisante n'a encore été trouvée, joue aussi un rôle. La formation postgraduée a un coût qui doit être rétribué dans une mesure raisonnable. Or il n'est pas imaginable que cette rétribution, une fois versée par les cantons aux hôpitaux, disparaisse dans les caisses des hôpitaux. Non, cet argent doit revenir aux différentes divisions qui disposent d'une offre de formation structurée. Nous y veillerons.

En déclarant qu'«une bonne formation postgraduée, ça coûte de l'argent, une mauvaise encore davantage», un des participants à la Journée a exprimé avec pertinence l'importance pour notre système de santé de compter sur des médecins compétents, au bénéfice d'une formation postgraduée diversifiée et approfondie leur conférant des bases solides pour prendre les mesures et les décisions nécessaires. Sans eux, les débats toujours animés sur les critères EAE (efficacité, adéquation, économicité) n'auraient aucun sens. En matière de santé, ce sont en effet les médecins qui continueront demain de piloter le navire.

«Une bonne formation postgraduée, ça coûte de l'argent, une mauvaise encore davantage."

La réflexion selon laquelle l'engagement en faveur de la qualité de la formation prégraduée, postgraduée et continue a un impact immédiat sur la qualité des soins, dépasse largement les frontières de notre pays. Peter Rubin, ancien président du Conseil de l'Ordre des médecins anglais, affirmait: «Medical education is the bedrock on which high-quality health care is built. Teaching is important but education is vital.» Il ne reste plus qu'à conclure en ajoutant qu'il nous appartient de trouver les moyens et les méthodes pour que le transfert de connaissances et de savoir-faire aux jeunes médecins redevienne pour tous une mission normale, valorisante et reconnue. 